\section{Multiple-organ autoimmunity}

SIR - Autoimmune diseases affecting the thyroid, parathyroids, adrenals, stomach and pancreatic islets are genetically associated ${ }^{1}$ and patients with a disease affecting one of these organs show an increased prevalence of diseases affecting others, as do their close relatives. This observation has given rise to the rather obvious suggestion that some patients may produce autoantibodies that recognize antigenic determinants which are common to two or more of these organs ${ }^{2,3}$. Recent studies, reported in Nature, showing that virallyinduced monoclonal antibodies in mice sometimes cross-react with several organs have given renewed prominence to this explanation.

We have carried out extensive absorption studies to determine whether the simultaneous occurrence of two or more auto-immune diseases in one patient can be explained simply by autoantibodies which cross-react with the different organs involved. Sera containing autoantibodies reactive with two autoantigens were absorbed with one of those antigens and then tested for residual activity against that antigen and also against the other. The combinations tested were: thyroid microsomal autoantibody (TMaab) and gastric parietal cell autoantibody (PCaab); thyroid stimulating autoantibody (TSaab) and TMaab; thyroglobulin autoantibody (TGaab) and TMaab. In none of these cases could we find the cross-reaction predicted; thus the autoantibody does not explain co-occurrence of these diseases.

This does not, however, indicate that cross-reactions between multiple tissue antigens never occur. On the contrary, there is striking evidence that some of the autoantibodies observed in systemic lupus erythematosus (SLE) recognize antigenic determinants occurring in a variety of seemingly unrelated molecules has provided a great simplification of a disease which was formerly bewildering ${ }^{4}$.

Further evidence against the simple cross-reaction explanation is the discordance in time of onset of multiple autoimmune diseases in individual patients $^{1}$. If cross-reactivity were the explanation, one might expect that in patients with two autoimmune diseases, both would appear simultaneously; this is not usually the case. Thus in only 10 per cent of 113 patients with both Addison's disease and insulin-dependent diabetes did both diseases present simultaneously; the average interval in the remainder was nearly 6 years $^{5}$. Similarly, in a series of patients with pernicious anaemia and Graves' disease, the mean interval between diagnosis of the two conditions was 3.3 years with a range of $0-9$ years $^{6}$. These observations point to the likely role of somatic mutation in generating the immune diversity required for reaction with these diverse autoantigens and suggest that the genetic influence must act on precursors of the pathogenic clones rather than on the clones themselves.

Advocates of "disordered immune regulation" notwithstanding, it seems obvious that specific autoimmune diseases must be a consequence of an unfortunate clonal specificity within an individual's immune response repertoire ${ }^{7}$. On this view, individuals not genetically predisposed to a particular disease will either have deleted the precursor of a particular forbidden clone or will possess anti-idiotypic clones capable of deleting the forbidden clones as they arise.

A corollary of this view is that in patients with multiple autoimmune diseases, the pathogenic clones are likely to have arisen by separate somatic mutations from a common precursor, or from separate precursors which share idiotypic determinants ${ }^{1}$, thereby explaining why such diseases do not usually appear simultaneously. Sharing of idiotypic determinants between antibodies directed against closely related antigens seems most probable even though cross-reaction is not apparent in the paratopes of such antibodies ${ }^{1}$. We predict that this is the key to understanding the occurrences of multiple autoimmune diseases in individual patients.

JOHN G. KNIGHT ALLISON KNIGHT

Otago University Medical School,

PO Box 913 ,

Dunedin, New Zealand

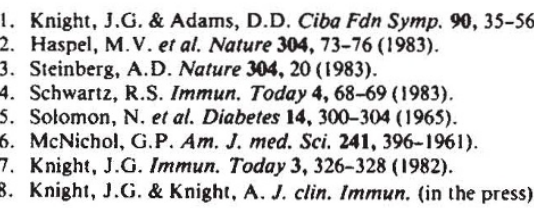

\section{Supernovae and life}

SIR - Rubenstein et al. ${ }^{1}$ have suggested that optical synchrotron emission from supernovae might be substantially circularly polarized. However, the optical emission from the Crab Nebula has $<1 \%$ circular polarization ${ }^{2,3}$, and there are simple reasons for believing that naturally occurring synchrotron emission from extremely relativistic electrons will have a very low degree of circular polarization.

The synchrotron emission from an electron is confined within a cone, approximately aligned with its instantaneous direction of motion, and having a halfwidth of $\sim\left(m c^{2}\right) / E$ (rad). For electrons emitting in the visible, even if the field is as high as $10 \mathrm{G},\left(m c^{2}\right) / E \approx 10^{-3}$. The emission from a single electron is $100 \%$ polarized linearly polarized near the axis of the emission cone, $\mathrm{RH}$ elliptically polarized on the side of the cone towards the direction of magnetic field, and LH elliptically polar- ized on the opposite $\operatorname{side}^{4}$. At the halfpower points the degree of circular polarization is about 0.4 .

The synchrotron emission from a group of relativistic electrons will have these same polarization properties only if the "pitch angles" of the spiral motions of the electrons all lie within a range which is less than the width of the emission cone of a single electron - that is, $<\left(m c^{2}\right) / E$. This condition is evidently satisfied for electrons in accelerators where considerable care is taken to ensure that the electron pitch angles are closely equal to $90^{\circ}$. It is unlikely to be true in supernovae.

When the range of electron pitch angles is large compared with the width of the individual emission cones, the radiation emitted in a particular direction comes almost equally from the $\mathrm{RH}$ and $\mathrm{LH}$ polarized sides of individual emission cones. The circularly polarized component is thus largely cancelled and the resulting degree of circular polarization becomes ${ }^{5}$ $0\left(m c^{2}\right) / E$.

In summary, if synchrotron emission is to be substantially circularly polarized the pitch angles of the electrons must be confined within a range less than the width of the emission cone of individual electrons: this includes the special case discussed by Epstein ${ }^{6}$. For extremely relativistic electrons the emission cone is so small that it is unlikely that this condition will be met in astronomical objects. Substantial circular polarization can arise when the electrons are barely relativistic and the emission is at low harmonics of the gyro-frequency (" "gyro-synchrotron", emission). Since strong magnetic fields are believed to result when stars collapse, gyrosynchrotron emission might possibly provide Rubenstein et al. with a source of circularly polarized radiation in supernovae.

J. A. ROBERTS

Division of Radiophysics, CSIRO,

PO Box 76,

Epping, NSW 2121, Australia

1. Rubenstein, E. Bonner, W.A. Noyes, H.P. \& Brown, G.S. Nature 306, 118 (1983).

2. Landstreet, J.D. \& Angel, J.R.P. Nature 230, 103 (1971).

3. Martin, P.G., Illing, R. \& Angel, J.R.P. Mon. Not. R. astr. Soc. 159, 191-201 (1972).

4. Korchak, A.A. \& Syrovatskii, S.I. Astr. Zh. 38, $885-897$ (1961); Soviet Astr. -AJ 5, 678-686 (1962).

S. Legg. M.P.C. \& Westfold, K.C. Astrophys. J. 154, 499-514 (1968).

6. Epstein, R.I. Astrophys. J. 183, 593-610 (1973).

7. Chanmugam, G. \& Dulk, G.A. Astrophys. J. 244, $569-578$ (1981).

\section{Scientific Correspondence}

Scientific Correspondence is intended to provide a forum in which readers may raise points of a rather technical character which are not provoked by articles or letters previously published (where Matters Arising remains appropriate). 